\title{
STABILITY OF A NEW, MULTICHANNEL, LOW-LEVEL LIQUID SCINTILLATION COUNTER SYSTEM, KVARTETT
}

\author{
SIGURDUR EINARSSON \\ Multi-Detector Systems Ltd., Skúlatún 6, 105 Reykjavík, Iceland \\ and \\ PÁLL THEODÓRSSON
}

Science Institute, University of Iceland, Dunhaga 3, IS-107 Reykjavík, Iceland

\begin{abstract}
Kvartett is a new liquid scintillation counting (LSC) system for radiocarbon dating that takes a radical departure from conventional systems to obtain a compact, low-level counting system measuring four samples simultaneously. Each sample vial, inside the well of a large NaI(Tl) guard-counter crystal (facing down), sits on top of a vertical PMT. The fourfold counting capacity can be used to increase the number of samples being dated or to get higher precision. The increased throughput helps to keep a rigid quality-control standard. We monitored the background count rate almost continuously for 7 months, and measured the count rate of a standard repeatedly for 2 months. The results show the background and system reproducibility to be stable.
\end{abstract}

\section{INTRODUCTION}

Over the last 15 years, many radiocarbon dating laboratories have participated in intercomparison measurements. The results of these intercomparison studies show that an alarming number underestimated their uncertainty figures. This can probably be explained by the long counting times required for controlling the stability and precision of the counting systems which, inevitably, compromises the volume of sample throughput. To get sufficient statistical accuracy in determining the activity of low-level samples, one must count a sample for at least a day, which severely limits the counting capacity of the liquid scintillation counting (LSC) system. To mitigate this problem, multidetector systems were developed for solid and gaseous samples in ${ }^{14} \mathrm{C}$ dating (Schoch et al. 1980; Theodórsson 1988). The concept of multidetector systems has been transferred to LSC in a new low-level system, Kvartett. Although this system was designed primarily for ${ }^{14} \mathrm{C}$ dating, it can equally well be used for all $\beta$ emitters except tritium, where low background cannot be obtained because low-energy $\beta$ particles will give pulses where the photo tube noise is great. A stable counting efficiency and background are the prime requirements of an LSC system for ${ }^{14} \mathrm{C}$ dating. We have studied the two parameters thoroughly with the new system and report the results here.

\section{DESCRIPTION OF KVARTETT}

Background measurements with a set of gas proportional counters under different active and passive shields have demonstrated convincingly that an active guard consisting of a 102 -mm-thick annular $\mathrm{NaI}(\mathrm{Tl})$ scintillation detector (Loosli, Forster and Otlet 1986) gives the best active shield. This can be explained by the fact that the $\mathrm{NaI}(\mathrm{Tl})$ detector not only eliminates the muon background component but also largely suppresses the background contribution of the secondary gamma rays produced by energetic cosmic muons and protons in the thick passive shield surrounding the sample detector. Thus, we used a NaI(Tl) guard counter for our four-sample LSC system. To contain the cost of the $\mathrm{NaI}$ (Tl) crystal, we used the old single photomultiplier tube (PMT) system, which was almost completely abandoned in the late 1950s in favor of the more complicated two-tube systems working in coincidence to suppress the thermal pulses of the tubes. With refined production methods, it has been possible to decrease drastically the dark pulses of the PMTs. Further, a Chinese low-level LSC 
system, giving very promising results, has been reported (Pei-yun and Ting-kui 1983). We selected $29 \mathrm{~mm}$ diameter PMTs to keep the size of each detector as small as possible.

The specially made conical ( 3 or $5 \mathrm{ml}$ ) quartz vial sits on the front face of a vertical PMT. The vials are wrapped with thin Teflon ${ }^{\otimes}$ tape for maximum light reflection. The four sample detectors are closely packed, and during counting, the four vials are enclosed by the large $(152 \mathrm{~mm} \times 102 \mathrm{~mm})$ $\mathrm{NaI}(\mathrm{Tl})$ well-type crystal.

The samples sit on a special tray that can be changed automatically by first lowering the tray and then rotating the carousel $90^{\circ}$. This system can take up to 4 trays or 16 samples.

A $40 \mu \mathrm{Ci}^{241} \mathrm{Am}$ standard can be brought automatically into the center of the vial tray for energy calibration. The photo peak at $60 \mathrm{keV}$ provides an easy method for energy calibration. Each sample channel has its own HV supply, amplifier and multichannel analyzer (MCA) that splits the spectra into an anticoincidence and a coincidence spectrum (1024 channels in each) depending on the absence or presence of a simultaneous pulse from the $\mathrm{NaI}(\mathrm{Tl})$ guard counter.

A personal computer accepts data from the four sample channels and the guard detector. A Microsoft $^{\circledR}$ Windows ${ }^{\mathrm{TM}}$ based, menu-driven program (KAP) allows detailed data processing and easy access to counting parameters. Counting data are updated at the end of every period. A filing system permits storage and retrieval of counting parameters, counting data and spectra. The program is controlled through pull-down menus. Counting parameters are displayed in tabular form and modifications are entered in spreadsheet fashion. Statistical information on the current counting process can be displayed for all channels and time-series graphs of periodic counting data can be displayed for individual channels together with a statistical summary of the data. At the end of the counting process, a report can be printed with average and individual-period cpm, expected uncertainty and standard deviation. A table with gross counts for every period and a time-series plot can be printed in the report. The spectrum display shows one channel at a time, which is selected through one of the menus.

Counting data are stored on disk at the end of each period, including sample cpm, counting time and other relevant data. Calibration figures are also stored every time the external source is used for calibration. Standard and background measurements are kept in separate files where data accumulate on individual vials and counters. Data in these files can be accessed using commands from the menu. Background and standard measurements are treated differently from samples. Vials have different background and light transmission characteristics and thus need to be independently calibrated. Periodic data are stored in the same way as before, but new data are accumulated over extended periods for individual vials. In this way, long-term data are made available for individual vials, and statistical control graphs can easily be constructed for quality assurance.

\section{METHODS}

We measured the system background in 50-min intervals using splits of the same background sample for all counting channels. The background sample is a commercially obtained analytical-grade benzene with $15 \mathrm{~g} \mathrm{liter}^{-1}$ butyl-PBD. The vials are dedicated to the same counting channel throughout the measurements as some variability exists between them. We made 4630 measurements in each counting channel over a period of 7 months.

To study counting reproducibility, we prepared a sample from a commercial ${ }^{14} \mathrm{C}$ benzene standard and added butyl-PBD to obtain a concentration of $15 \mathrm{~g} \mathrm{liter}^{-1}$. The activity of the sample was $c a .1 .2$ 
DPS $\mathrm{g}^{-1}$. We used a different batch of quartz vials for the reproducibility measurements, but each vial was dedicated to the same counting channel as before. Due to the high count rate of the sample, we chose 20 -min counts. We made 230 measurements in two months in each counting channel.

We used a $10 \mu \mathrm{Ci}$ external ${ }^{241} \mathrm{Am}$ source to calibrate the Kvartett. The peak appeared at $60 \mathrm{keV}$ (Fig. 1), and we adjusted the counting window from 25 to $156 \mathrm{keV}$. We used the external source on a regular basis to check the system calibration throughout the measurements.

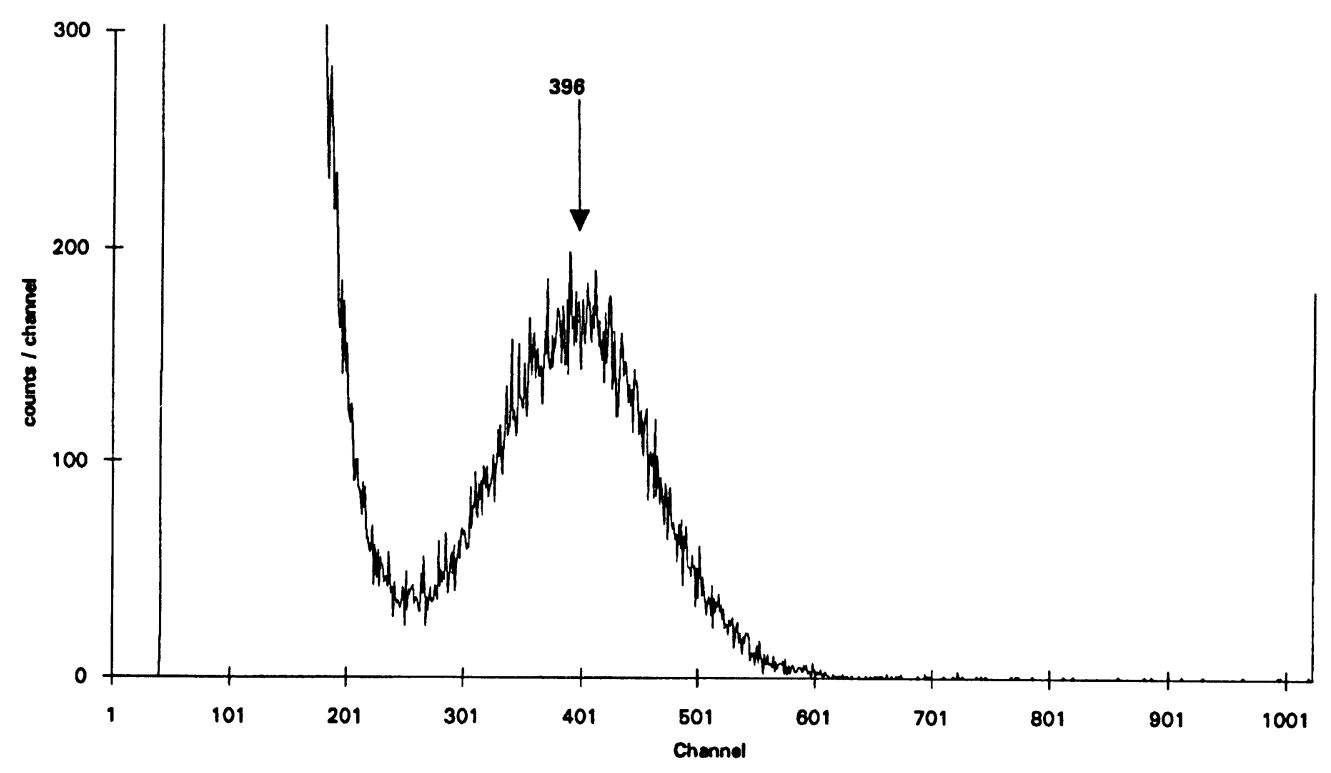

Fig. $1 .{ }^{241} \mathrm{Am}$ spectrum in Kvartett. The $60 \mathrm{keV}$ peak appears in channel 396.

A common method to demonstrate system stability is to produce time-series graphs of background and standard sample measurements. This is an informal method that does not explicitly take into account the overall variability of the results. Switsur (1990) suggested the use of statistical quality control graphs or charts as a form of quality assurance in radiocarbon dating. These control charts are based on a study of the variability of results, and to construct them, measurements are divided into groups of equal size. Different charts are used to study different features of the measurements, e.g., long and short-term drift, instability. The sensitivity of the graphs to detect drift increases with subgroup size. Time and budget, however, limit the number of background and standard measurements in the laboratory thus limiting the size of the subgroup. For this study, we chose a subgroup size of ten for both background and reproducibility measurements. The three types of statistical control charts are: 1 ) the mean $(\bar{x})$ chart constructed by plotting the mean of each group against the group number; 2 ) the range (R) chart where the range of the means from the grand mean is plotted against the group number; 3 ) and the standard deviation $(\sigma)$ chart where the standard deviation for each group is plotted against the group. The groups are arranged in chronological order. Based on the analysis of the variability, upper and lower control limits are calculated and plotted in the charts where $99.8 \%$ of the samples will lie within them. Any drift or instability will immediately become apparent as trends in the data or as an excessive number of outliers. In this study, we use the $\bar{x}$ and the $\sigma$ control charts. 


\section{Counting Efficiency}

To measure the counting efficiency of the system, we weighed $4.1678 \pm 0.0005 \mathrm{~g}$ of benzene, synthesized from SRM-4990C oxalic acid (HOxII) into a quartz vial at the Scottish Universities Research and Reactor Centre (SURRC). We measured the standard in 20-min counts 51 times. The counting window represented an energy range from 25 to $156 \mathrm{keV}$ and the counting efficiency was $65.85 \pm 0.16 \%$.

\section{RESULTS}

\section{Counting Statistics}

The counting process is generally expected to be governed by Poisson statistics. Various factors in the counting process may, however, introduce other sources of error, which include benzene purity and vial reproducibility (Pearson 1979). We used the same vial and benzene sample throughout, practically eliminating these factors from consideration. However, one must consider that variability due to atmospheric pressure causes fluctuations in the cosmic background. The $\mathrm{NaI}(\mathrm{Tl})$ guard is an efficient anticoincidence detector that can probably reduce this effect considerably.

To examine the Poisson nature of the measurements (Cook et al. 1992) we look at the sampling distribution of $\mathrm{p}$, which has the following definition (Anderson, Sweeney and Williams 1994)

$$
\mathrm{p}=(\mathrm{n}-1) \mathrm{s}^{2} / \mathrm{\sigma}^{2}
$$

where $\mathrm{s}^{2}$ is the sample variance, $\sigma^{2}$ is the population variance (or the Poisson variance) and $n$ is the sample size. Assuming a Poisson distribution, the variance is equal to the grand mean and $p$ will have an approximate chi-squared distribution. According to theory, the value of $p$ should then lie within the limits set by the chi-squared distribution. For the background measurements, the data from channel 4 were divided into groups of ten and values for $p /(n-1)=s^{2} / \sigma^{2}$ obtained. Figure 2 shows a plot of $\mathrm{p} /(\mathrm{n}-1)$ against the groups in chronological order. The limits, UCL and LCL, indicated in the figure correspond to a $99.8 \%$ confidence limit according to the values of chi-squared obtained from the chi-squared table divided by $(n-1)$. A line through $p /(n-1)=1$ is shown in the graph to represent the case of $s=\sigma$.

A few points lie outside the upper and lower control limits of the distribution. A point lying outside the upper control limit indicates excessive oscillations inside the group. Figure 1 shows three such points. On the other hand, points lying outside the lower control limit show less variability where the standard deviation is much smaller than the expected value. In general, however, the evidence indicates that the data are Poisson distributed.

Figure 3 shows a similar plot for the reproducibility measurements. The data were divided into groups of ten and the value of $\mathrm{p} /(\mathrm{n}-1)$ plotted against the groups in chronological order. The upper control limits (UCL) and lower control limits (LCL), indicated in the figure, correspond to a $99.8 \%$ confidence limit according to the values of chi-squared obtained from the chi-squared table, divided by $(n-1)$. A line is also drawn through $\mathrm{p} /(\mathrm{n}-1)=1$ as before. From the graph in Figure 3, no evidence suggests that the data distribution deviates from Poisson behavior.

\section{Background Variability}

To demonstrate background variability we produced $\bar{x}$ and $\sigma$ control charts for the data from one of the counting channels. For each group of ten values, we calculated a mean and standard deviation. 
For the $\bar{x}$ chart the mean values are plotted against the groups in chronological order as shown in Figure 4. To evaluate the grand mean, we used the following definition.

$$
\mu=(1 / \mathbf{k}) \Sigma \mathbf{x}_{\mathbf{i}}
$$

where $\mathrm{k}$ is the total number of groups and $\mathrm{x}_{\mathrm{i}}$ is the mean of the ith group. A line is drawn to represent the grand mean. Two more lines are drawn to represent the LCL and UCL, calculated using the following definition

$$
\begin{aligned}
& \mathrm{LCL}=\mu-\mathrm{A} \sigma \\
& \mathrm{UCL}=\mu+\mathrm{A} \sigma
\end{aligned}
$$

where $\mu$ is the grand mean, $\sigma$ is the expected standard deviation and $A$ is obtained from tables (Beyer 1968). Ca. $99.6 \%$ of the group mean values should lie within these limits.

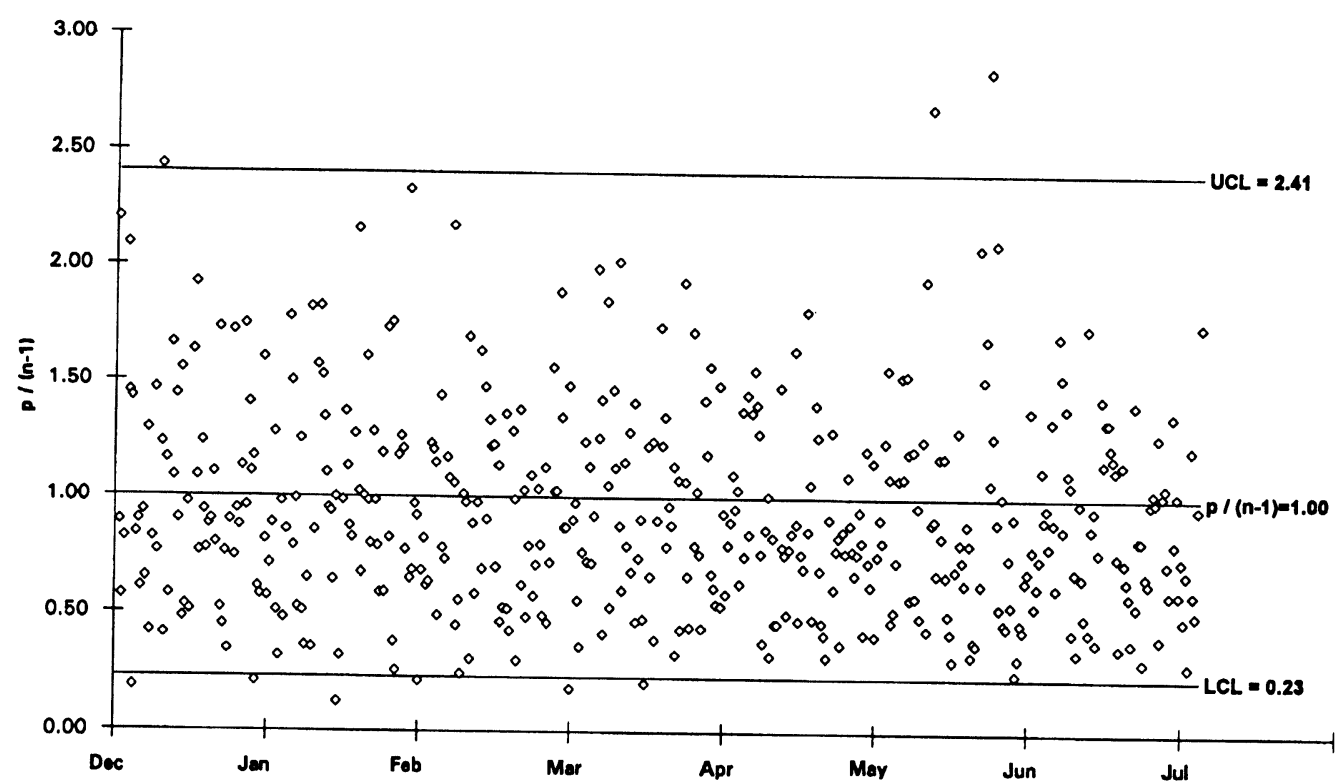

Fig. 2. A plot of $\mathrm{s}^{2} / \sigma^{2}$ against the group in chronological order for the background.

Examining the $\bar{x}$ chart for the background, we make two observations: 1) 2 out of the 460 samples are outside the control limits, which corresponds to $c a$. $0.4 \%$ of the total; 2) a small-scale drift can be detected from the distribution of the group means. This may be due to variations in atmospheric pressure for the 7-month period when the data were collected (Fig. 4). However, one would expect this variation to be very small due to the high efficiency of the $\mathrm{NaI}(\mathrm{Tl})$ guard counter in removing the cosmic background. Examining the total background for this counter (i.e., system background + the events vetoed by the $\mathrm{NaI}(\mathrm{Tl})$ guard), larger-scale variation are observed, but following the same general pattern. This would tend to suggest that the drift is due to variations in atmospheric pressure, but to a much smaller degree in the system background due to the $\mathrm{NaI}(\mathrm{Tl})$. 


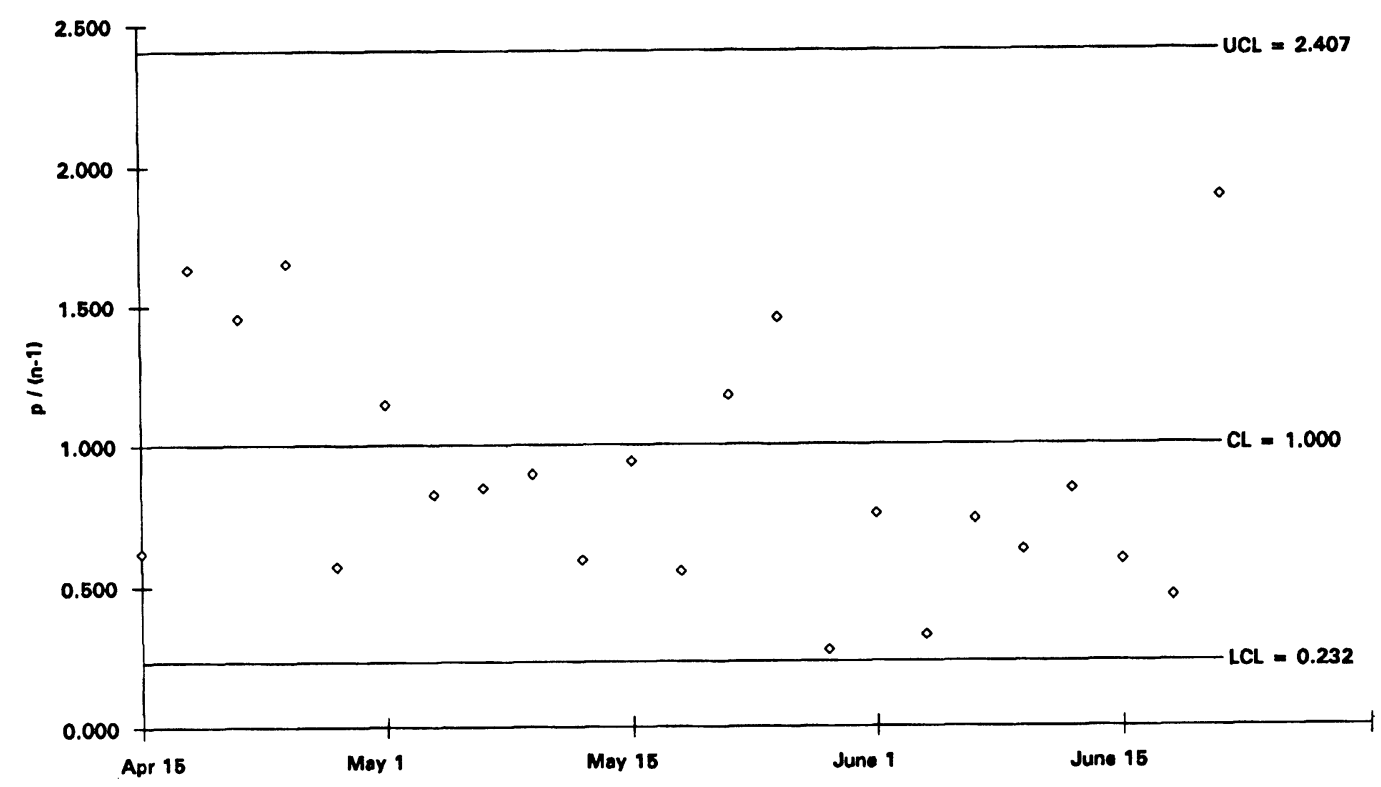

Fig. 3. A plot of $\mathrm{s}^{2} / \sigma^{2}$ against the group in chronological order for the stability measurements

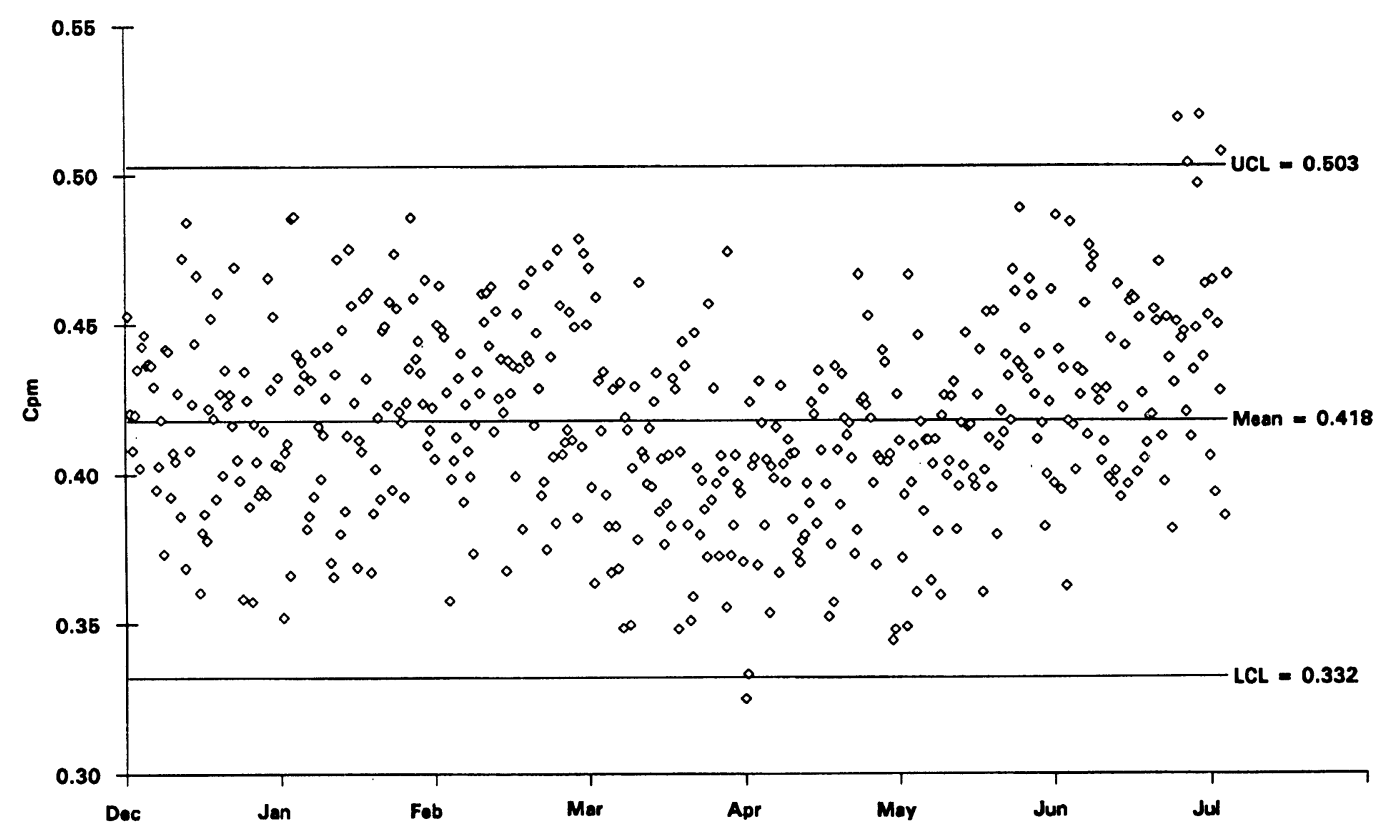

Fig. 4. $\bar{x}$ control chart of system background over 7 months

We constructed the $\sigma$ chart by plotting the standard deviation of the group means against the groups in chronological order as shown in Figure 5. The variability of the counting process can be assessed by drawing a set of lines representing the UCL and LCL where $c a .99 .6 \%$ of the standard deviation 
points should lie. The points are not symmetrical about the mean standard deviation, and according to Beyer (1968), the central line (CL) for the $\sigma$ graph has the following definition

$$
\mathrm{CL}=\mathrm{c}_{2} \mathrm{~s}_{\mathrm{p}}
$$

where $c_{2}^{\prime}$ is obtained from tables (Beyer 1968) and $s_{p}$ is the pooled standard deviation. The UCL and LCL are calculated using

$$
\begin{aligned}
\mathrm{LCL} & =\mathrm{B}_{2}{ }^{\prime} \mathrm{s}_{\mathrm{p}} \\
\mathrm{UCL} & =\mathrm{B}_{4}{ }^{\prime} \mathrm{s}_{\mathrm{p}}
\end{aligned}
$$

where $B_{2}^{\prime}$ and $B_{4}^{\prime}$ are obtained from tables (Beyer 1968).

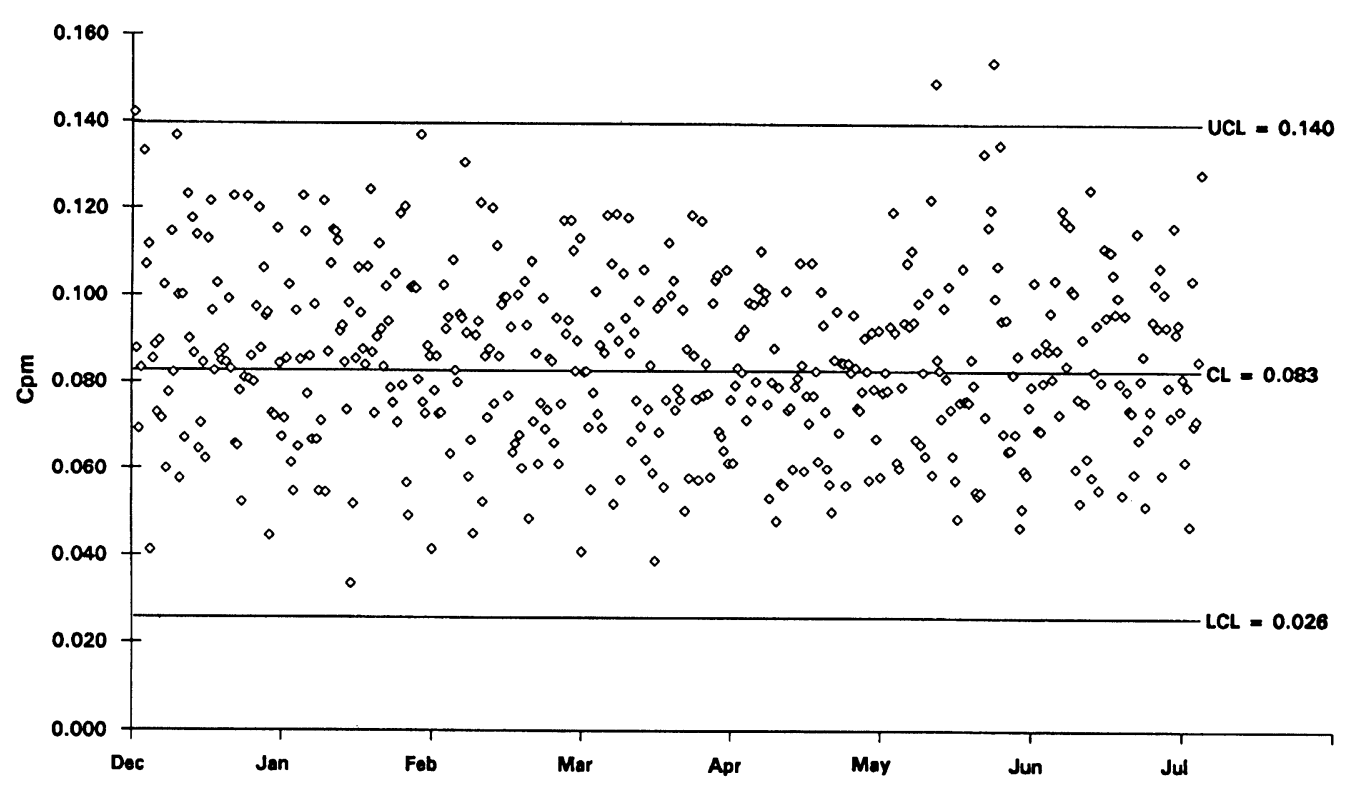

Fig. 5. $\sigma$ control chart for the background over a 7-month period

The $\sigma$ chart shows no apparent drift or excessive fluctuations apart from two values that lie outside the upper control limit. These correspond to two of the standard deviation values lying outside the UCL in Figure 1. Re-examining the original samples for the first of these data points, we found the excessive fluctuation to be due to one excessive 50-min count. For the second point, the excess fluctuation is due to the individual counts distributed in the extremes either above or below the expected mean. In the first case, one could eliminate the single bad count and accept a new mean and standard deviation for the remaining samples. In the second case, one would tend to discard the whole group. Apart from these two excessive values, the system reveals no indication of large- or small-scale drift in the distribution.

\section{${ }^{14} \mathrm{C}$ Measurements}

We use the counts obtained for the ${ }^{14} \mathrm{C}$ sample in a channel to demonstrate the variability of the counting process in Kvartett. We divide the measurements into groups of ten and find the mean and standard deviation for each group. We then plot the mean values against the groups in chronological order in the $\bar{x}$ chart shown in Figure 6, and calculate the grand mean using equation (2). We use 
Equations (3) and (4) to calculate the UCL and LCL. We observed no excessive variability in the samples from the ${ }^{14} \mathrm{C}$ measurements shown in Figure 6 . The variability of the standard deviation is assessed in the $\sigma$ chart in Figure 7.

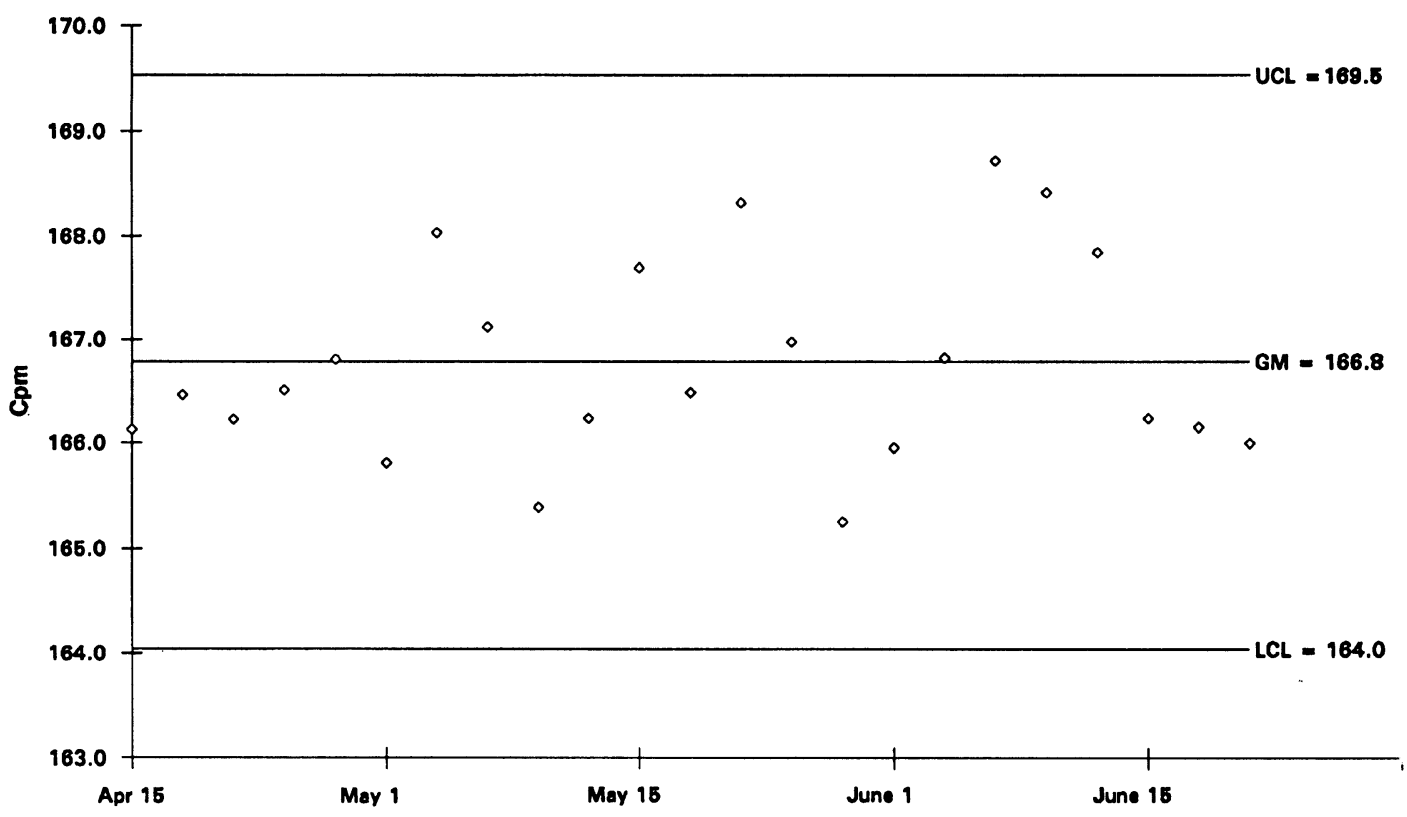

Fig. 6. $\bar{x}$ control chart showing the ${ }^{14} \mathrm{C}$ measurements over a 2-month period

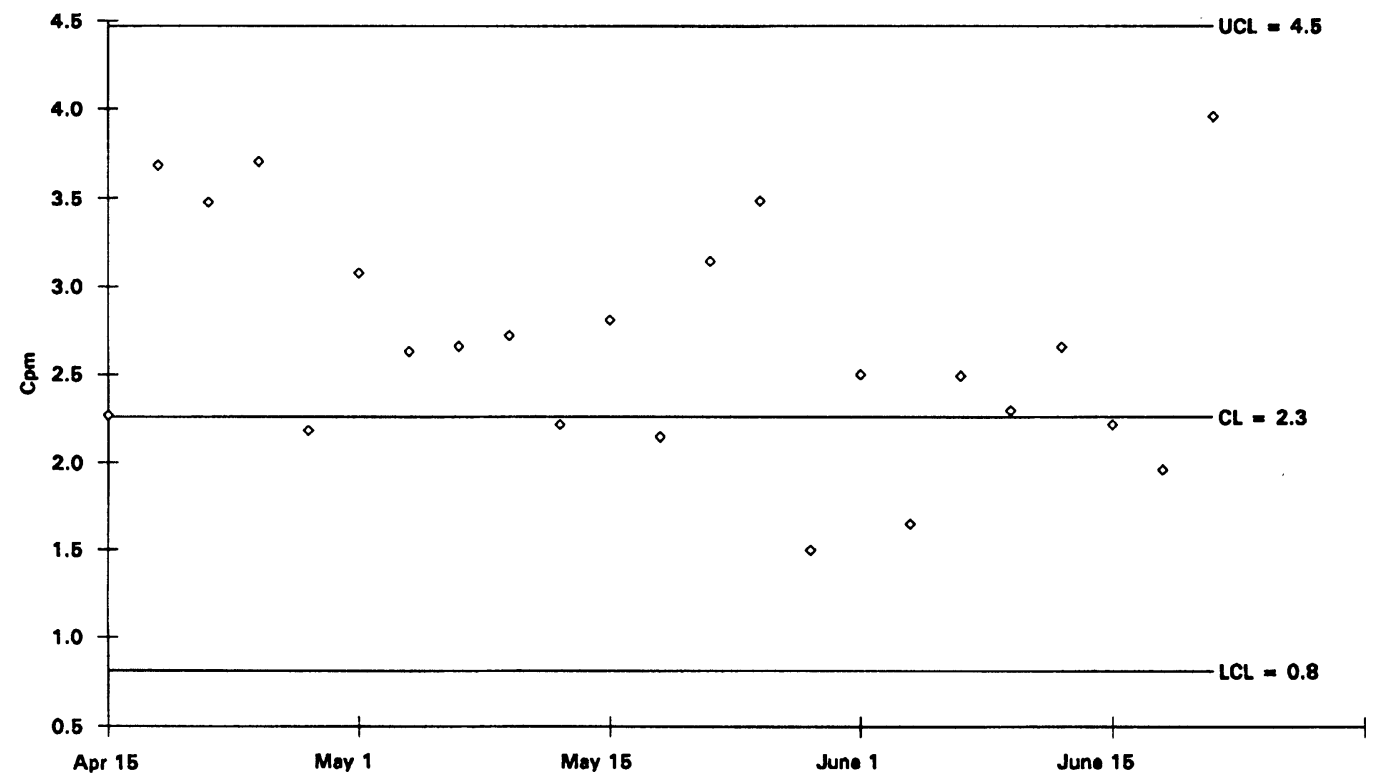

Fig. 7. $\sigma$ control chart for the standard deviation of the ${ }^{14} \mathrm{C}$ measurements 
We constructed the $\sigma$ chart using the standard deviations calculated for the group means as before. Equations (6) and (7) were then used to calculate the LCL and UCL. Lines representing the limits are shown in the graph. We calculated the central line using Equation (5).

No evidence of excessive variability of the data or any small- or large-scale trends can be detected in the $\sigma$ chart for the standard deviation of the ${ }^{14} \mathrm{C}$ sample.

\section{DISCUSSION AND CONCLUSION}

We examined the background in Kvartett over a 7-month period and the reproducibility of the counting process for 2 months. The measurements show the background to be stable within the UCL and LCL in the $\bar{x}$ and $\sigma$ control graphs, although a small-scale drift appears in the background mean, possibly due to atmospheric pressure variations. Correction for atmospheric variations removed any
evidence of fluctuations.

The distribution of the measurements of the ${ }^{14} \mathrm{C}$ sample in the $\bar{x}$ control chart in Figure 6 stay well within the control limits, indicating that the system is stable. No evidence of drift in the standard deviation control chart in apparent in Figure 7, where all the data are inside the control limits. Therefore, we conclude that the system reproducibility is stable during these measurements.

The counting efficiency for a counting window representing an energy range from 25 to $156 \mathrm{keV}$ is $\sim 66 \%$. It is possible to lower the counting window to at least $16 \mathrm{keV}$, thus increasing the counting efficiency to $\mathrm{ca}$. 75\%. However, the background increases, and may increase $>2$ times for a window of 16-156 keV (Einarsson 1992). Using four independent counting channels in a single instrument increases the sample throughput and allows more time for background and standard measurements. Many data points may be collected this way and statistical control charts can be constructed with grı ster accuracy. This is the basis for good quality assurance in any situation.

The ternal standard used in Kvartett forms the basis of the energy calibration. Measuring the 60 $\mathrm{keV}$ peak oifers a powerful method to monitor the calibration and hence the system stability. Frequent checks by inserting the source between counting periods allows the tracking of system calibration during the counting process. This is an important factor in high precision We intend to continue monitoring the background and sample reproducibility in the Kvartett. We are also planning to measure counting efficiency and background for tritium.

\section{REFERENCES}

Anderson, D. R., Sweeney, D. J. and Williams, T. A. 1994 Introduction to Statistics Concepts and Applicatiuns. Minneapolis/St. Paul, West Publishing Company: $413 \mathrm{p}$.

Beyer, W. H., ed. 1968 Handbook of Tables for Probability and Statistics. Boca Raton, Florida, CRC Press: $642 \mathrm{p}$.

Cook, G. T., Scott, E. M., Wright, E. M. and Anderson, R. 1992 The statistics of low-level counting using the new generation of Packard liquid scintillation counters. In Long, A., and Kra, R. S., eds., Proceedings of the 14th International ${ }^{14} \mathrm{C}$ Conference. Radiocarbon 34(3): 360-365.

Einarsson, S. A. 1992 Evaluation of a prototype lowlevel liquid scintillation multisample counter. In Long, A., and Kra, R. S., eds., Proceedings of the 14th Inter- national ${ }^{14} \mathrm{C}$ Conference. Radiocarbon 34(3): $366-$ 373.

Loosli, H. H., Forster, M. and Otlet, R. L. 1986 Background measurements with different shielding and anticoincidence systems. In Stuiver, M. and Kra, R. S., eds., Proceedings of the 12 th International ${ }^{14} \mathrm{C}$ Conference. Radiocarbon 28(2A): 615-662.

Pearson, G. W. 1979 Precise ${ }^{14} \mathrm{C}$ measurement by liquid scintillation counting. Radiocarbon 21(1): 1-21.

Pei-yun, F. and Ting-kui, Z. 1983 A single photomultiplier liquid scintillation counting apparatus for ${ }^{14} \mathrm{C}$ low-level measurement. In McQuarrie, S. A., Ediss, C. and Wiebe, L. I., eds., Advances in Scintillation Counting. Edmonton, Canada, University of Alberta: 456-467.

Schoch, H., Bruns, M., Münnich, K. O. and Münnich, M. 
1980 A multicounter system for high precision ${ }^{14} \mathrm{C}$ measurements. In Stuiver, M. and Kra, R. S., eds., Proceedings of the 10 th International ${ }^{14} \mathrm{C}$ Conference. $R a$ diocarbon 22(2): 442-447.

Switsur, R. 1990 A consideration of some basic ideas for quality assurance in radiocarbon dating. In Scott, E. M., Long, A. and Kra, R. S., eds., Proceedings of the
International Workshop on Intercomparison of Radiocarbon Laboratories. Radiocarbon 32(3): 341-346.

Theodórsson, P. 1988 Development of multicounter systems for low-level beta samples. In Garcia-Leon, M. and Madurga, G., eds., Low-level Measurements and their Applications to Environmental Radioactivity. Singapore, World Scientific Publishing: 459-470. 